\title{
Obesità sarcopenica
}

\author{
Edda Parrinello ${ }^{1} \cdot$ Lorenzo M. Donini $^{1}$
}

Accettato: 25 maggio 2020 / Pubblicato online: 26 ottobre 2020

(c) The Author(s) 2020

Sommario L'obesità sarcopenica è una sindrome più frequentemente presente nella popolazione anziana, associata ad aumentato rischio di disabilità, morbilità e mortalità. Questo articolo discute l'evoluzione e le controversie legate alla definizione di tale patologia, i fattori di rischio, i meccanismi fisiopatologici, le conseguenze clinico-funzionali, le modalità di valutazione della composizione corporea e i criteri diagnostici presenti. Vengono descritte, inoltre, le possibili strategie di trattamento.

Parole chiave Obesità · Sarcopenia $\cdot$ Obesità sarcopenica . Composizione corporea

\section{Definizione, prevalenza e fisiopatologia}

Per obesità sarcopenica s'intende una condizione clinicofunzionale caratterizzata da un'alterazione della composizione corporea in cui si verifica un eccesso di massa grassa e un deficit di massa muscolare (sarcopenia). Attualmente non esiste una definizione univoca di tale patologia in termini di criteri diagnostici e cut-off; per questo motivo, non è tuttora possibile determinarne la prevalenza precisa, né l'effettiva rilevanza clinica e funzionale. La prevalenza stimata varia infatti dal 2,75 a oltre il $20 \%$ [1].

Proposta da Lorenzo M. Donini.

Materiale elettronico supplementare La versione elettronica di questo articolo (https://doi.org/10.1007/s40619-020-00789-x) contiene materiale supplementare, disponibile per gli utenti autorizzati.

$\varangle$ E. Parrinello

eddaparrinello@hotmail.it

1 Dipartimento di Medicina Sperimentale, Sapienza Università di Roma, Roma, Italia
Nell'obesità sarcopenica, la fisiopatologia della sarcopenia e quella dell'obesità sono fortemente interconnesse. Come regola empirica, l'evidenza suggerisce che Fat Free Mass (FFM) e Fat Mass (FM) siano soggette alla cosiddetta one quarter rule: per qualsiasi incremento del grasso corporeo, si verifica una variazione parallela di FFM, corrispondente approssimativamente al $25 \%$ [2]. La perdita di massa e funzione muscolare può inoltre essere favorita dall'obesità come fattore di rischio indipendente, a causa delle alterazioni ad essa correlate: l'infiammazione cronica di basso grado, l'aumento dello stress ossidativo, l'insulino-resistenza (con diminuzione della capacità anabolica della muscolatura striata, oltre al peggioramento del profilo glucidico), lo stile di vita sedentario e la maggiore incidenza di malattie croniche che possono avere un impatto negativo sul catabolismo muscolare [3]. L'infiammazione cronica di basso grado, presente nell'obesità e da essa favorita, determina una diminuzione della funzione anabolica muscolare, mediata dalle variazioni della produzione di fattori quali TNF, IL-6, leptina, $\mathrm{GH}[4]$.

Studi recenti dimostrano che l'adiposità, soprattutto quella di tipo viscerale, determina alterazioni dell'asse GH/Insulin Like Growht Factor 1 (IGF 1) in grado di favorire il depauperamento muscolare, indipendentemente da età e sesso [5]. Sia l'invecchiamento che l'obesità favoriscono, inoltre, l'accumulo di tessuto adiposo in sede ectopica, in particolare a livello del fegato, del midollo osseo e del muscolo scheletrico, sostenendo fenomeni di lipotossicità e infiammazione. A livello intramuscolare il grasso si accumula prevalentemente tra i diversi gruppi muscolari, delimitato dalle fasce. Viene descritto con l'acronimo IMAT (Intra-Muscolar Adipose Tissue) e può essere quantificato mediante risonanza magnetica nucleare (MRI). La presenza di IMAT può promuovere alterazioni della differenziazione delle cellule mesenchimali progenitrici, compromettendo il 


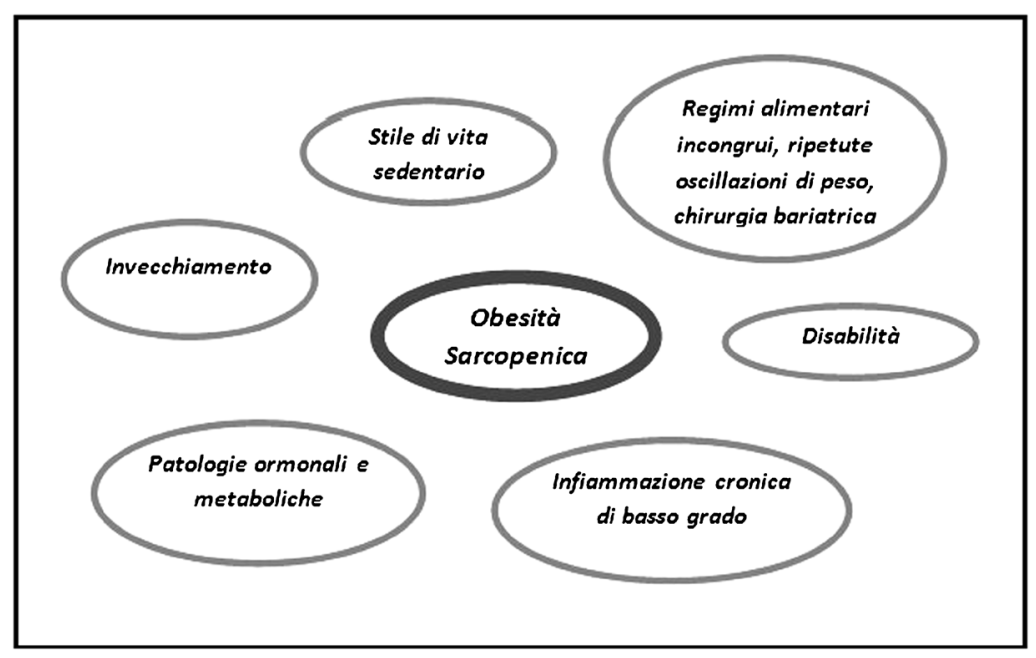

Fig. 1 Fattori di rischio per l'obesità sarcopenica. Tra i maggiori fattori di rischio per obesità sarcopenica troviamo il fisiologico invecchiamento, lo stile di vita sedentario e la disabilità, regimi alimentari incongrui, ripetute oscillazioni di peso con depauperamento della massa muscola- re, talvolta interventi di chirurgia bariatrica, soprattutto se non seguiti da idonei regimi alimentari, l'infiammazione cronica di basso grado e le patologie endocrino-metaboliche come il diabete mellito di tipo 2, l'insulinoresistenza, l'ipogonadismo e l'ipercortisolismo potenziale rigenerativo del muscolo e stimolando l'instaurazione di processi di fibrosi. Gli adipociti a livello muscolare inoltre secernono ormoni paracrini e citochine proinfiammatorie che promuovono un ciclo di feedforward producendo lipidi intra-miocellulari. Questa lipotossicità compromette la contrattilità delle fibre muscolari e interferisce con la sintesi proteica muscolare, esacerbando la sarcopenia $[6,7]$. La deposizione lipidica può verificarsi anche in spazi precedentemente occupati dai muscoli, compromettendo la crescita del nuovo tessuto muscolare. Uno studio di Manini e collaboratori, ad esempio, ha riportato un aumento della deposizione di lipidi intramuscolari dopo che uomini e donne giovani e sani di età compresa tra 19 e 28 anni erano stati esposti a 30 giorni di inutilizzo delle gambe, con conseguente perdita di massa muscolare degli arti inferiori [8].

\section{I fattori di rischio e le conseguenze cliniche}

L'obesità sarcopenica è una condizione maggiormente rappresentata nella popolazione geriatrica. La tendenza a sviluppare sarcopenia con l'età è legata alla fisiologica graduale riduzione della massa muscolare che si verifica già dalla terza decade di vita. La sarcopenia età-correlata è però da differenziare dalla cachessia o muscle wasting, termini con i quali s'intende il depauperamento della massa magra non legate all'età bensì ad altre patologie di natura infiammatoria cronica, neoplastica, distrofica o infettiva (es. epatite C). I cambiamenti della composizione corporea legati all'età sono in parte attribuibili a modificazioni ormonali, come la diminuzione di estrogeni e testosterone. Le donne in menopausa tendono a diminuire la propria massa magra e aumentare la grassa, con particolare propensione all' accumulo di grasso viscerale. Negli uomini, il testosterone promuove la rigenerazione muscolare e la sintesi proteica, pertanto la sua diminuzione con l'età può mettere a rischio la massa muscolare [9].

L'obesità sarcopenica non colpisce, però, esclusivamente la popolazione geriatrica: può infatti essere presente in pazienti obesi con fattori di rischio quali condizioni acute di malattia, disabilità, patologie croniche come scompenso cardiaco, patologie endocrinologiche e metaboliche (sindrome metabolica, dislipidemie, insulino-resistenza, deficit di GH, ipogonadismo). Altre condizioni che possono predisporre allo sviluppo di obesità sarcopenica sono inadeguati regimi dietetici (soprattutto in caso di rapide e ripetute perdite di peso, seguite da incremento ponderale con inadeguato recupero della massa magra) e la chirurgia bariatrica, in particolare quando non seguita da un'adeguata supervisione nutrizionale [4, 7]. I fattori di rischio per lo sviluppo di obesità sarcopenica sono illustrati in Figura 1.

Diversi studi dimostrano che le condizione di obesità e di sarcopenia peggiorino la prognosi di svariate patologie (neoplastiche, insufficienze d'organo) ed è possibile che l'obesità sarcopenica comporti un rischio ancora superiore, derivato dall'effetto cumulativo delle due singole condizioni. È stata dimostrata l'associazione tra obesità o, soprattutto, obesità sarcopenica e scarse prestazioni fisiche (vedi $E d$ monton Obesity Staging System, EOSS). Le conseguenze a lungo termine della perdita di massa muscolare scheletrica sembrano peggiori nei soggetti con obesità, in cui si sviluppa uno squilibrio relativo tra la massa grassa in eccesso e il deficit di massa magra, anche quando i livelli di massa muscolare scheletrica sono conservati in valore assoluto [4].

Numerosi lavori hanno inoltre osservato una correlazione tra obesità sarcopenica e aumentato rischio di ospedalizza- 


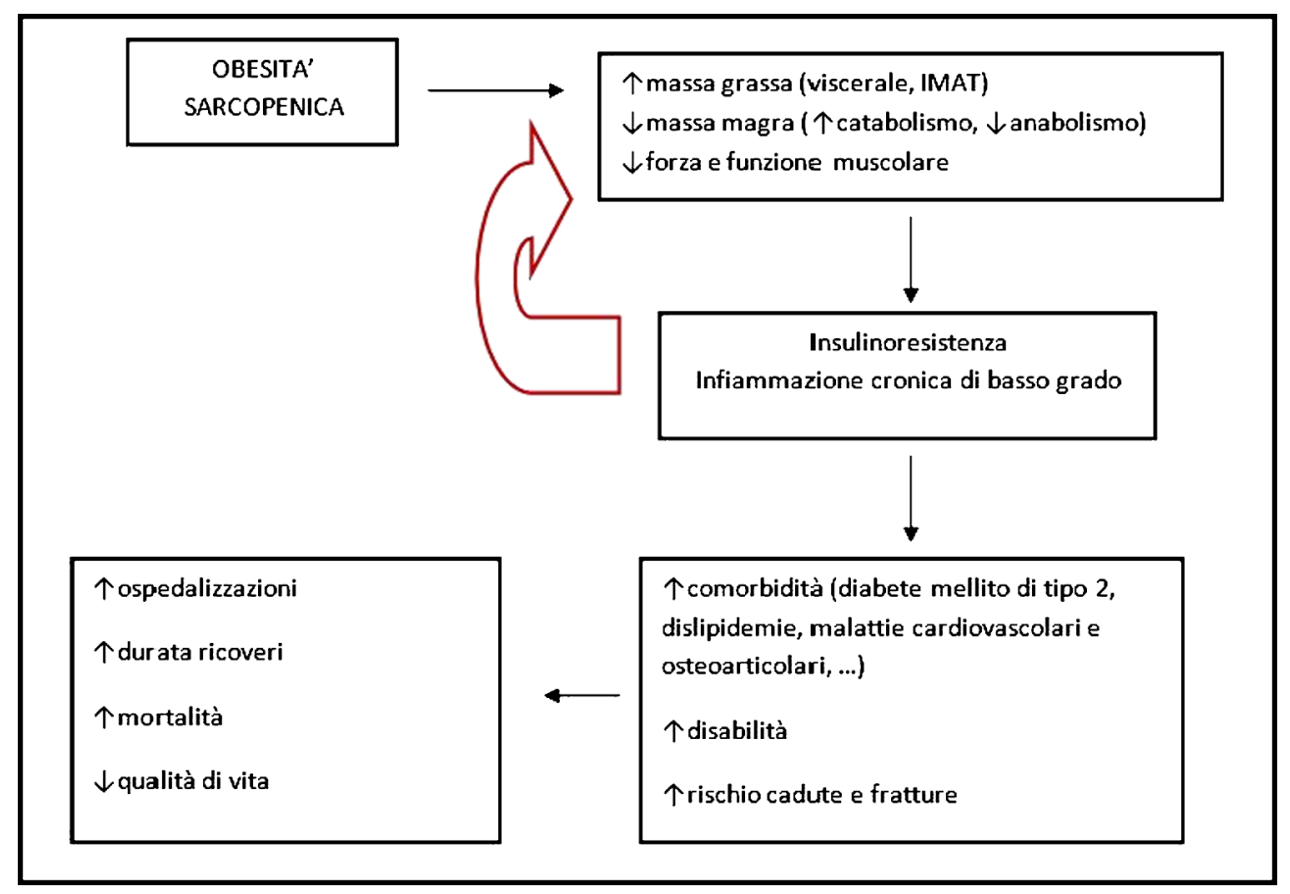

Fig. 2 Conseguenze fisiopatologiche dell'obesità sarcopenica. L'aumento della massa grassa e la diminuzione della massa muscolare, tipici dell'obesità sarcopenica, determinano insulinoresistenza e infiammazione cronica di basso grado che, da un lato, alimentano le alterazioni della composizione corporea tipiche della patologia stessa e,

zioni e di mortalità per tutte le cause. Altri studi segnalano associazioni con altre condizioni patologiche come la NonAlcoholic Fatty Liver Disease (NAFLD), in cui l'associazione con la sarcopenia è stata dimostrata indipendentemente dalla presenza di obesità e insulinoresistenza, carenza di vitamina D, osteoporosi, osteoartiriti, sintomi depressivi e diminuzione della qualità della vita [1], come rappresentato in Figura 2.

\section{Diagnosi}

Una questione di fondamentale importanza per la diagnosi di obesità sarcopenica è l'individuazione di una definizione univoca: alcuni studiosi definiscono tale patologia come coesistenza di obesità e sarcopenia (valutate separatamente), altri la considerano come un'entità a sé stante, sulla base del fatto che l'aumento della massa grassa e la diminuzione della magra interagiscano sinergicamente creando un fenotipo distinto. In quest' ultimo caso diventa necessario valutare l'obesità sarcopenica attraverso parametri che considerino contemporaneamente le due condizioni, ad esempio il rapporto tra FM e FFM, o il rapporto tra Visceral Fat Area (VFA) e Thigh Muscle Area (TMA) valutati mediante TC. Attualmente in letteratura è più estesamente utilizzata una valutazione separata delle due condizioni (Tabella 1), mentre la definizione unificata è usata raramente. dall'altro, determinano un aumento del rischio di comorbidità e disabilità. Ne consegue un aumento delle ospedalizzazioni e della mortalità $\mathrm{e}$, in generale, un peggioramento della qualità di vita del paziente con obesità sarcopenica. IMAT, Intra-Muscolar Adipose Tissue

Tabella 1 Parametri più utilizzati in letteratura per definire la sarcopenia e l'obesità. $A S M$, appendicular skeletal mass $(\mathrm{kg}) ; W t$, weight $(\mathrm{kg})$; $h$, height (m); $B M I$, body mass index; $F F M$, fat free mass (\%); $F M$, fat mass (\%); $G S$, gait speed (m/s); HGS, hand grip strength (kg); VFA, visceral fat area $\left(\mathrm{cm}^{2}\right) ; W C$, waist circumference; EWGSOP, European Working Group for the Study of Sarcopenia; FNIH, Foundation for the National Institutes of Health

\begin{tabular}{ll}
\hline Sarcopenia & Obesità \\
\hline ASM $/ \mathrm{Wt}$ & BMI \\
$\mathrm{ASM} / \mathrm{h}^{2}$ & FM \\
$\mathrm{ASM} / \mathrm{h}^{2}$ plus GS e/o HGS (EWGSOP) & WC \\
$\mathrm{FFM} / \mathrm{h}^{2}$ & VFA \\
$\mathrm{ALM} / \mathrm{BMI}$ e HGS (FNIH) &
\end{tabular}

La comunità scientifica sta ancora considerando la possibilità di includere all'interno della definizione anche la riduzione della forza muscolare (dinapenia). Uno dei metodi più diffusi per la valutazione della forza muscolare è il cosiddetto handgrip strength test, facile e veloce da eseguire. L'inclusione di questo parametro è da molti incoraggiato, considerando che la riduzione della handgrip strength in pazienti geriatrici è correlata a un' aumentata incidenza di morbilità e mortalità, soprattutto in ambito cardiovascolare. È possibile, inoltre, che il calo della forza muscolare avvenga prima e a una velocità maggiore del vero e proprio depauperamento muscolare [10]. 
La stessa sarcopenia è definita in maniera eterogenea e diversi sono i parametri utilizzati a livello internazionale, dalla FFM alla Appendicular Lean Mass (ALM), usati in valore assoluto o normalizzati per il peso, l'altezza o, più raramente, il Body Mass Index (BMI). Lo European Working Group for the Study of Sarcopenia (EWGSOP) e la Foundation for the National Institutes of Health (FNIH) includono nell'algoritmo diagnostico anche parametri funzionali come hand grip strength o gait speed $[11,12]$.

Per quanto riguarda la definizione di obesità, l'American Association of Clinical Endocrinology raccomanda l'uso delle soglie di massa grassa (FM), ma considera anche l'utilizzo di parametri quali circonferenza vita (come indicatore del grasso viscerale) e BMI, seppur quest'ultimo presenti importanti limiti legati alla mancanza di specificità nella valutazione qualitativa della composizione corporea [13].

Tutti i metodi utilizzati per la valutazione della composizione corporea mostrano alcuni limiti (antropometria, DEXA, BIA, MRI, CT). La Dual-Energy X-ray Absorptiometry (DXA) è raccomandata sia per la valutazione della massa magra appendicolare nella diagnosi di sarcopenia, sia per quella della massa grassa e del tessuto adiposo viscerale, essendo un esame conveniente, disponibile e accurato [11]. L'ampia disponibilità è assicurata dal suo utilizzo in ambito di screening per osteoporosi e, inoltre, l'esame correla bene con le misure gold standard per la composizione corporea, come la risonanza magnetica. La Body Impedence Assessment (BIA) è uno strumento diagnostico di semplice esecuzione, non invasivo, economico, rapido e portatile. Tuttavia, la valutazione corporea può essere poco affidabile a causa dell'interferenza di alcuni fattori: le possibili alterazioni del tessuto muscolare, lo stato di idratazione del paziente e la forma del suo corpo [14]. Tecniche come la risonanza magnetica (MR) o la tomografia assiale computerizzata (TAC) sono sicuramente più precise e affidabili, ma più costose e meno accessibili; potrebbero quindi essere utilizzate in ambito di ricerca e come gold standard di riferimento, o in ambito clinico nel caso in cui il paziente abbia già a disposizione tali esami diagnostici a causa di altre condizioni.

\section{Possibili strategie preventive e terapeutiche}

Gli interventi sullo stile di vita, come la restrizione calorica e l'attività fisica sono le principali strategie di prevenzione e trattamento dell'obesità sarcopenica, potendo impedire o rallentare i processi fisiopatologici alla base dello sviluppo dell'obesità sarcopenica.

Il lavoro di Villareal e collaboratori mostra come in individui con obesità sarcopenica la perdita di peso o l'esercizio fisico da soli migliorino la funzione fisica e la combinazione dei due abbia un'azione ancora superiore [15]. Il rischio della restrizione calorica è quello della perdita di massa magra, oltre che di massa grassa (circa un quarto della massa magra per unità di peso, che potrebbe favorire la sarcopenia e la demineralizzazione ossea) [16].

$\mathrm{Al}$ momento, nessun intervento dietetico specifico è stato testato per il trattamento dell'obesità sarcopenica, ma si è osservato che strategie che ottimizzano l'anabolismo proteico durante la perdita di peso, come l'assunzione di proteine prima dell'esercizio fisico (più ancora di un'assunzione distribuita lungo tutto l'arco della giornata) possa prevenire la sarcopenia indotta dalla perdita di peso. Inoltre, la fonte di proteine, $i$ tempi di assunzione e specifici aminoacidi possono avere un ruolo importante per favorire un aumento della massa e della forza muscolare. L'esercizio fisico può influire sull'equilibrio ormonale, indurre la sintesi mitocondriale, modulare la funzione immunologica e migliorare la capacità ossidativa muscolare. L'aumento della sintesi proteica indotto dall'esercizio fisico sensibilizza il muscolo all'azione dell'insulina, promuovendone l'anabolismo. È stato osservato che l'esercizio aerobico, l'allenamento di resistenza e la loro combinazione riducono i biomarcatori infiammatori circolanti (tra cui IL-6, proteina C-reattiva e TNF) e determinano un aumento della sintesi proteica muscolare negli anziani, nonostante la riduzione degli stimoli anabolici legata all'età [4].

Senza dubbio sono necessarie altre ricerche per stabilire con precisione il ruolo di diversi aspetti nell'obesità sarcopenica: la combinazione dei macronutrienti all'interno di una dieta, il tipo di proteine da somministrare (siero di latte, caseina), i tempi di assunzione in relazione all'esercizio fisico, la composizione specifica di aminoacidi essenziali (ruolo di leucina o creatina) e l'eventuale integrazione di vitamina D e calcio.

Alcuni trattamenti innovativi sono in fase di studio, ad esempio la periodizzazione dell'esercizio fisico anche per pazienti sedentari, anziani o fragili, o la terapia con testosterone e modulatori selettivi del recettore degli androgeni, il cui razionale sarebbe l'aumento della massa muscolare e ossea, l'aumento del IGF-1 e la diminuzione dei marker infiammatori, ma i dati in merito al loro impatto sulla forza e funzione muscolare sono ancora contrastanti [17]. La metanalisi di Corona e colleghi (2016) dimostra gli effetti positivi del testosterone sulla composizione corporea, sul metabolismo glucidico e lipidico e sulla perdita di peso [9].

Tra i trattamenti farmacologici in via di sperimentazione, ricordiamo l'anamorelina, un analogo della grelina, usato in ambito oncologico negli stati di cachessia neoplastica, che potrebbe migliorare l'anabolismo muscolare e lo stato infiammatorio [4].

\section{Conclusione}

Una recente revisione sistematica della letteratura ha dimostrato la profonda inadeguatezza della ricerca disponibile 
sull'obesità sarcopenica in termini di coerenza di definizione, criteri diagnostici e strumenti metodologici, mettendo in evidenza la grande eterogeneità dei dati presenti in letteratura. I risultati di tale lavoro non consentono infatti conclusioni definitive [1]. Tale variabilità rappresenta sicuramente un grande ostacolo sia in ambito clinico che di ricerca e un numero di esperti sempre maggiore auspica al raggiungimento di un consensus, in quanto l'assenza di omogeneità è considerata uno dei principali ostacoli all'avanzamento delle conoscenze in merito a tale patologia. L'identificazione di una definizione condivisa e di precisi criteri diagnostici sarebbe di grande utilità per indicare una metodologia diagnostica gold standard e dei surrogati più facilmente riproducibili, per omologare la gestione del paziente nella pratica clinica e per individuare le migliori strategie di prevenzione e trattamento, alla luce del fatto che i dati epidemiologici esistenti, sebbene parzialmente discordanti, indichino un'elevata prevalenza di obesità sarcopenica e delle sue conseguenze clinico-funzionali. Le crescenti sfide associate all'obesità sarcopenica, infatti, probabilmente peggioreranno con il cambiamento della distribuzione demografica della nostra popolazione che invecchia. Terapie efficaci basate sull'evidenza potrebbero essere utili per migliorare la funzione fisica negli anziani e la loro qualità di vita, oltre a prevenirne l'ospedalizzazione.

Funding Note Open access funding provided by Università degli Studi di Roma La Sapienza within the CRUI-CARE Agreement.

Conflitto di interesse Gli autori Edda Parrinello e Lorenzo M. Donini dichiarano di non avere conflitti di interesse.

Consenso informato Lo studio presentato in questo articolo non ha richiesto sperimentazione umana.

Studi sugli animali Gli autori di questo articolo non hanno eseguito studi sugli animali.

Nota della casa editrice Springer Nature rimane neutrale in riguardo alle rivendicazioni giurisdizionali nelle mappe pubblicate e nelle affiliazioni istituzionali.

Open Access This article is licensed under a Creative Commons Attribution 4.0 International License, which permits use, sharing, adaptation, distribution and reproduction in any medium or format, as long as you give appropriate credit to the original author(s) and the source, provide a link to the Creative Commons licence, and indicate if changes were made. The images or other third party material in this article are included in the article's Creative Commons licence, unless indicated otherwise in a credit line to the material. If material is not included in the article's Creative Commons licence and your intended use is not permitted by statutory regulation or exceeds the permitted use, you will need to obtain permission directly from the copyright holder. To view a copy of this licence, visit http://creativecommons.org/licenses/by/4.0/.

\section{Bibliografia}

1. Donini LM, Busetto L, Bauer JM et al (2019) Critical appraisal of definitions and diagnostic criteria for sarcopenic obesity based on a systematic review. Clin Nutr 39(8):2368-2388

2. Gallagher D, DeLegge M (2011) Body composition (sarcopenia) in obese patients: implications for care in the intensive care unit. $\mathrm{J}$ Parenter Enteral Nutr 35:21

3. Cleasby ME, Jamieson PM, Atherton PJ (2016) Insulin resistance and sarcopenia: mechanistic links between common co-morbidities. J Endocrinol 229:R67-81

4. Batsis JA, Villareal DT (2018) Sarcopenic obesity in older adults: aetiology, epidemiology and treatment strategies. Nat Rev Endocrinol 14(9):513e37

5. Berryman DE, Glad CA, List EO, Johannsson G (2013) The GH/IGF-1 axis in obesity: pathophysiology and therapeutic considerations. Nat Rev Endocrinol 9:346-356

6. Consitt LA, Bell JA, Houmard JA (2009) Intramuscular lipid metabolism, insulin action, and obesity. IUBMB Life 61:47-55

7. Kalinkovich A, Livshits G (2017) Sarcopenic obesity or obese sarcopenia: a cross talk between age-associated adipose tissue and skeletal muscle inflammation as a main mechanism of the pathogenesis. Ageing Res Rev 35:200-221

8. Manini TM, Clark BC, Nalls MA et al (2007) Reduced physical activity increases intermuscular adipose tissue in healthy young adults. Am J Clin Nutr 85:377-384

9. Corona G, Giagulli VA, Maseroli E et al (2016) Testosterone supplementation and body composition: results from a meta-analysis of observational studies. J Endocrinol Invest 39(9):967-981

10. McLeod M, Breen L, Hamilton DL, Philp A (2016) Live strong and prosper: the importance of skeletal muscle strength for healthy ageing. Biogerontology 17:497-510

11. Cruz-Jentoft AJ, Bahat G, Bauer J et al (2019) Writing group for the European working group on sarcopenia in older people 2 (EWGSOP2), and the extended group for EWGSOP2. Sarcopenia: revised European consensus on definition and diagnosis. Age Ageing 48(1):16e31

12. Studenski SA, Peters KW, Alley DE et al (2014) The FNIH sarcopenia project: rationale, study description, conference recommendations, and final estimates. J Gerontol, Ser A, Biol Sci Med Sci 69:547-558

13. Garvey WT, Mechanick JI, Brett EM et al (2016) American Association of Clinical Endocrinologists and American College of Endocrinology comprehensive clinical practice guidelines for medical care of patients with obesity. Endocr Pract 22(Suppl 3):1-203

14. Kyle UG, Bosaeus I, De Lorenzo AD et al (2004) Bioelectrical impedance analysis-part II: utilization in clinical practice. Clin Nutr 23:1430-1453

15. Villareal DT, Chode S, Parimi N et al (2011) Weight loss, exercise, or both and physical function in obese older adults. N Engl J Med 364:1218-1229

16. Heymsfield SB, Gonzalez MC, Shen W et al (2014) Weight loss composition is one-fourth fat-free mass: a critical review and critique of this widely cited rule. Obes Rev 15:310-321

17. Snyder PJ, Bhasin S, Cunningham GR et al (2016) Effects of testosterone treatment in older men. N Engl J Med 374:611-624 